Ragnar Nurk

\title{
EINE AUSSICHT AUF REVAL (TALLINN) SAMT IHRER BEFESTIGUNGEN
}

Wenn man heutzutage von der Anhöhe des Dombergs (estnisch: Toompea) in Richtung auf das Meeres hinüberblickt, sieht man hauptsächlich eine ebene Landschaft und einen Park mit Bäumen, die inzwischen hochgewachsen sind. Vor ungefähr anderthalb bis zwei Jahrhunderten sah hier alles gänzlich anderes aus. Rechts konnte man wohl auch damals die Unterstadt mit ihren Kirchtürmen und roten Dächern erblicken. Zu den Gemeinsamkeiten mit der heutigen Ansicht zählte, dass man auch die Stadtmauer mit ihren zahlreichen Türmen sah, welche die mittelalterliche, dicht bebaute Stadt umgibt - die kleineren vierkantigen Konsoltürme wechseln sich mit größeren Türmen mit einem dreiviertelkreisförmigen Grundriss ab. Aber was einem heute schwierig oder gar unmöglich vorstellbar ist, sind die späteren, neuzeitlichen Erdbefestigungen, die einen Teil der Zone der Bastionsbauten bildete, die ununterbrochen die gesamte Unter- und Oberstadt umgaben - wie man sieht, waren die Wälle zum Teil sogar doppelt angelegt. Die Vorstadt Fischermay (auch: Fischermai, estnisch: Kalamaja) am Meer hat wohl bis zum heutigen Tage ihr verhältnismäßig grünes Aussehen beibehalten, allerdings hat sie inzwischen eine etwas imposantere Bebauung erhalten. 


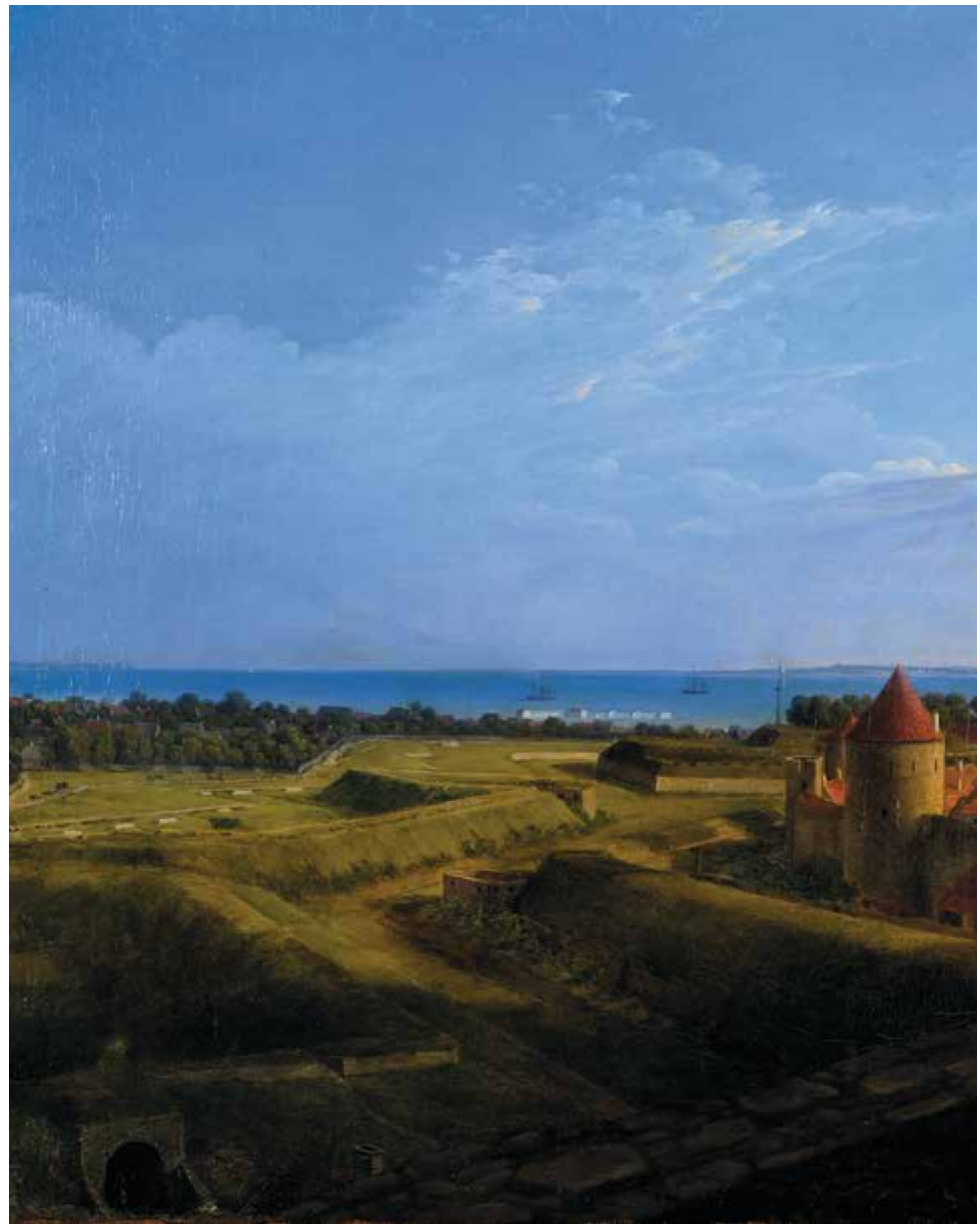

Abb. 1. Karl (Carl) Ferdinand von Kügelgen, „Eine Aussicht auf Reval von der Patkul-Treppe aus“, Ölgemälde, ca. 1830-1831, Estnisches Kunstmuseum 


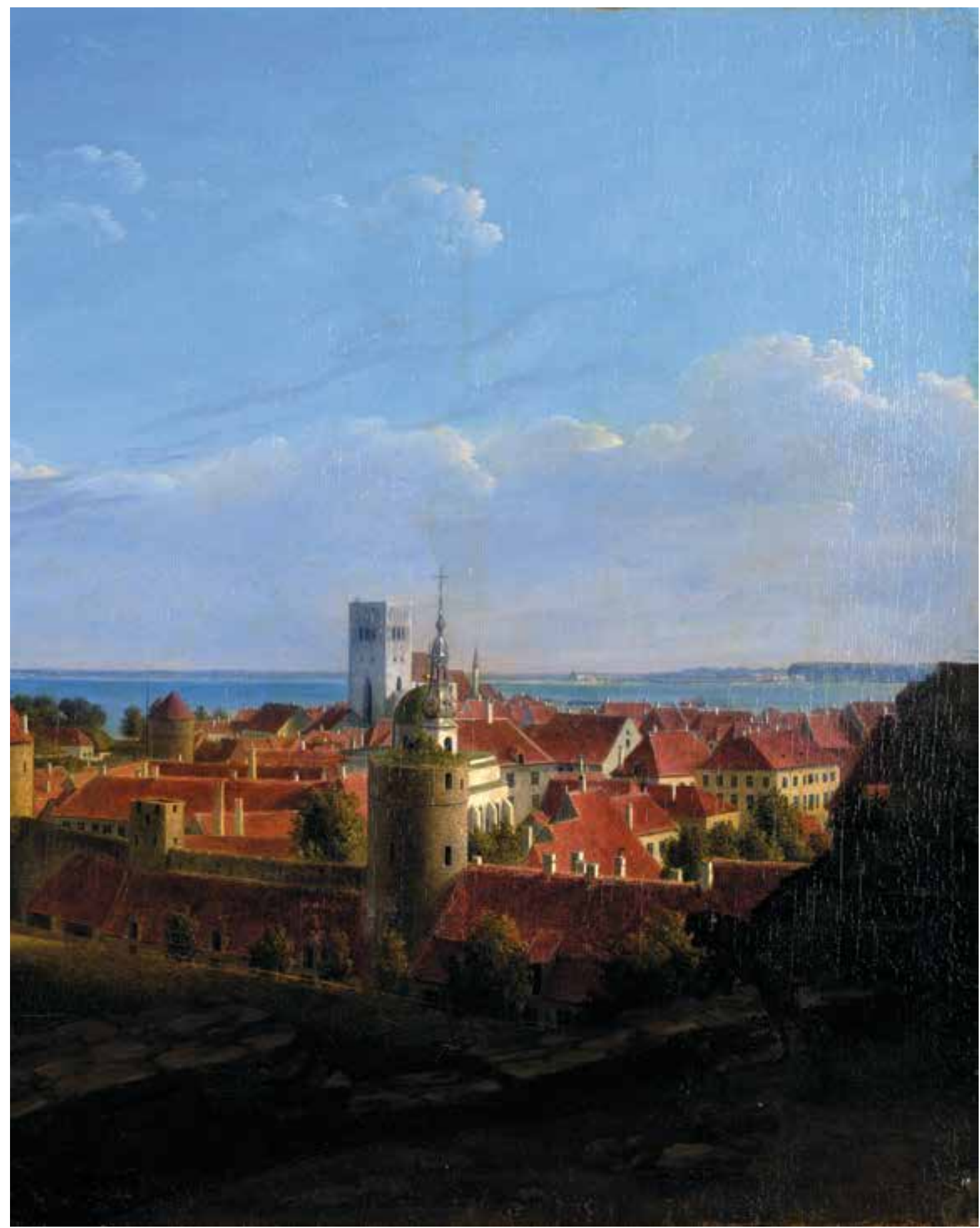




\section{ALLGEMEINES ÜBER DEN KÜNSTLER UND DAS GEMÄLDE}

Bei dem aus Rheinland stammenden Landschaftsmaler Karl (Carl) Ferdinand von Kügelgen (1772-1831) handelt es sich um einen der angesehensten Künstler, der je in Reval tätig gewesen war. Sein Zwillingsbruder Franz Gerhard von Kügelgen war ebenfalls ein Maler, spezialisiert auf Historienbilder und Porträts, der sich ebenso in Reval und im damaligen Russischen Reich aufhielt, allerdings für einen kürzeren Zeitraum. Die Brüder heirateten die Töchter eines deutschbaltischen Gutsherrn aus der unmittelbaren Nähe Revals. Der Stil Karl von Kügelgens entsprach gut der damals modischen klassizistischen Ästhetik, welche die Wirklichkeit etwas verschönerte und versuchte, das Zufällige beiseite zu lassen. Von Kügelgen stand im Dienst des kaiserlichen Hofes der Romanovs, er war Mitglied verschiedener Akademien der Künste und erfüllte die Aufträge der gehobenen Gesellschaftsschichten St. Petersburgs. Im Auftrage von Zar Alexander I. besuchte er sowohl die Krim als auch Finnland, das eben zum Bestandteil des Russischen Imperiums geworden war, um ihre Landschaften zu verewigen. Zusätzlich zu seinen Gemälden hat er Sepiazeichnungen und Lithographien angefertigt. Seit dem Jahre 1827 lebte er beständig in Reval, wofür er das Höfchen Friedheim mitsamt eines großen Gartens in der Vorstadt Antonius-Berg (estnisch: Tõnismäe) erwarb, um dort seinen Lebensabend zu verbringen. Von Kügelgen verstarb in den letzten Tagen des Jahres 1831 (nach dem alten Kalender) und wurde auf dem Friedhof der deutschen Gemeinde in der Nähe der Stadt in Ziegelskoppel (estnisch: Kopli) beigesetzt. ${ }^{1}$ Es wird angenommen, dass auch die zehn Gemälde zur Revaler Thematik, die uns als Originale oder aufgrund von Fotos bekannt sind, aus seinen letzten Lebensjahren stammen. Neun davon, die über ein gemeinsames Format

1 Dieser Abschnitt basiert auf: Voldemar Vaga, Kunst Tallinnas XIX sajandil (Tallinn: Kirjastus „Kunst”, 1976) 9-21; Hugo Peets, „Karl Ferdinand v. Kügelgen ja tema Tallinna-ainelised maalingud”, Vana Tallinn I (Tallinn: Tallinna Ajaloo Selts, 1936), 77-87. Der Sommergutshof Friedheim war in der Mitte des 18. Jahrhunderts eingerichtet worden. Das Gebiet des Höfchens ist später in eine große Zahl von Grundstücken aufgeteilt worden. In den zwanziger Jahren des 20. Jahrhunderts wurde ungefähr an die Stelle des früheren Hauptgebäudes ein neues errichtet, das höchstwahrscheinlich eine annähernde Kopie des alten Hauptbauwerks darstellt. Nach dem damaligen Besitzer, General Johan Laidoner, der im Freiheitskrieg (1918-1920) die estnische Armee angeführte hatte, ist das Gebäude bis heute unter dem Namen Laidoner-Villa bekannt. (Alo Särg, Tallinna mõisad ja mõisnikud (Tallinn: Argo, 2010), 37). Laut Hugo Peets war das Kreuz auf dem Grab von Kügelgens auch noch vor dem Zweiten Weltkrieg vorhanden. Der Friedhof wurde Anfang der fünfziger Jahre des 20. Jahrhunderts auf Befehl der Sowjetmacht vernichtet und an seine Stelle ein Park eingerichtet. 
verfügen, gehören einer Serie an. ${ }^{2}$ Es handelt sich um Ansichten der Stadt aus unterschiedlichen Richtungen mitsamt den sie umgebenden Naturlandschaften im Vordergrund und dem geräumigen Firmament über dem Horizont in der Ferne. Die Stadt stellt auf diesen Gemälden eher ein Element dar, das die Landschaft belebt. Das Ölgemälde, das im Zentrum des vorliegenden Artikels steht, „Eine Aussicht auf Reval von der Patkul-Treppe aus" ist dahingegen etwas größer (mit den Maßen von $56,5 \times 88 \mathrm{~cm}$ ). Seinen jetzigen Standort im Estnischen Kunstmuseum (Eesti Kunstimuuseum, EKM) erreichte es erst im Jahre 1952 und es kam aus dem Bestand des Staatlichen Russischen Museums (Gosudarstvennyj Russkij muzej, GRM) in St. Petersburg (damals Leningrad). Dem Gemälde fehlen eine Signatur und das Datum. Karl von Kügelgen hatte man das Gemälde bereits seitens des Russischen Museums zugeschrieben, aber es ist nicht bekannt, auf welcher Grundlage. Der Auftraggeber und auch das frühere Schicksal des Gemäldes, bevor es ins Museum geriet, sind ebenfalls unbekannt. Im Unterschied zu anderen Revalansichten von Kügelgens - zumindest zu denjenigen, die im Estnischen Kunstmuseum aufbewahrt werden - ist dieses Gemälde nicht auf Papier, sondern auf Leinwand gemalt. ${ }^{3}$

Nach Einschätzung der Kunsthistorikers Voldemar Vaga handelt es sich um eine verhältnismäßig außergewöhnliche Arbeit im Vergleich zu dem gesamten, uns bekannten Werk des Künstlers: „Das ist ein realistisches Meisterwerk. (...) Mit einer erstaunlichen Detailliertheit hat er auch die allerkleinsten Einzelheiten dargestellt, und das in ihrer ganzen Zufälligkeit. Das Gemälde ist aber nicht trocken, sondern wirkt sehr frisch und malerisch. (...) Der Reiz der Perspektive des Gemäldes ist unübertrefflich. Auf diesem Gemälde hat sich K. F. von Kügelgen auch als

2 Vaga, Kunst Tallinnas, 17.

3 Die jetzige Sammlungsnummer lautet M 2853. Das Gemälde hat man vom Russischen Museum in Leningrad zusammen mit einer anderen von Kügelgen signierten Ansicht Revals erhalten, Akt 419 (04.07.1952). Über die Autorenschaft gibt es auf der Arbeit nur Eintragungen hinter dem Unterrahmen im kyrillischen Alphabet offenbar aus Russischen Museum. Die Angaben über das Gemälde stammen von einer Kuratorin der Gemäldesammlung des Estnischen Kunstmuseums, Rita Kroon, 15.08.2013 und 19.08.2013. Das Gemälde ist in der digitalen Sammlung des Kunstmuseums vorhanden: http://digikogu.ekm.ee/ekm/search/oid-7681/?searchtype=simple\&searchtext=carl\%20 von\%20k\%C3\%BCgelgen\&offset=1 (abgerufen am 20.08.2013). Unter anderem ist das Gemälde auf der Ausstellung der Werke der Zwillingsbrüder, die im Jahre 1988 in Ost-Berlin stattgefunden hatte, ausgestellt worden (siehe die Broschüre Gerd-Helge Vogel, Malerei und Zeichnungen der Brüder Gerhard von Kügelgen (1772-1820) und Karl von Kügelgen (1772-1832) aus den Sammlungen der Museen in Tallin[n], Tartu und Leningrad (Berlin: Zentrum für Kunstausstellungen der DDR/Neue Berliner Galerie, 1989). 
ein großer Meister in Bezug auf die Darstellung der Luft und des Lichts gezeigt." Vaga vermutet, dass der Künstler, im Unterschied zu seiner gewöhnlichen Praxis, sich diesmal nicht mit flüchtigen, in der freien Natur angefertigten Skizzen zufriedengab, sondern direkt aus der Natur malte, offenbar von einem Fenster eines Hauses aus am Steilhang des Glints am Domberg. ${ }^{4}$ Auf dem gegebenen Gemälde steht die Stadt zweifelsohne im Mittelpunkt der Aufmerksamkeit. Das ist nicht ein Ausblick aus der Natur auf die Stadt, sondern ein Ausblick der Stadt selbst, der geschickt den von der Natur selbst angebotenen Blickwinkel ausnutzt. Der Kalksteinfelsen, der das Bild an einer Seite teilweise umrahmt, und der als Staffage eingesetzte schwarze Pudel wirken nicht unnatürlich. Die Vornehmheit des Motivs ist nicht gesucht, sondern einfach diesem Ort zu eigen. Gleichzeitig ist alles, was man sehen konnte, und teilweise erscheint sogar, dass das, was man nicht mit dem bloßen Auge hätte sehen können, mit einer sachlichen Genauigkeit wiedergegeben worden. Im Bild gibt es die dem Kügelgenschen Schaffen charakteristische Weiträumigkeit und Meisterschaft, aber wegen der Unterschiede zu seinen anderen Gemälden zum Thema Reval, die sowohl technischer Art sind als auch die Behandlungsweise betreffen, bleibt die Attribuierung zwangsläufig etwas fragwürdig. Zugleich ist es auch schwierig, einen anderen Künstler zu nennen, der zum angenommenen Zeitraum der Anfertigung des Gemäldes in Reval tätig gewesen wäre oder die Stadt besucht hätte und der fähig gewesen wäre, ein Werk auf solch hohem Niveau zu schaffen. Mehrere kleine Details auf dem Gemälde weisen weiterhin auf die hervorragende Ortskenntnis des Autors hin.

\section{HINWEISE ZUR DATIERUNG}

Für die Datierung der Aussicht von Karl Ferdinand von Kügelgen gibt es auf dem Bild mehrere Hinweise, wenn man annimmt, dass das Dargestellte weitgehend der Realität entsprochen haben könnte.

Als Erstes sticht der steinerne Teil des Turmes der Olaikirche (estnisch: Oleviste kirik), der ohne Spitzhelm ist, ins Auge. Es handelte sich um eine der zwei großen Kirchspielkirchen in Reval, in deren Falle man annimmt, dass sie ihren Anfang von einem Sakralbau der skandinavischen Kaufleute nahm, der sich im nördlichen Teil der Stadt befunden hatte.

4 Vaga, Kunst Tallinnas, 19. 
Die Kirche fing im Jahre 1820 (wieder einmal) nach einem Blitzschlag Feuer und brannte von innen komplett aus. Bis das Bild gemalt wurde, waren laut dem Dargestellten alle Dächer der Kirche außer dem Spitzhelm wiederaufgebaut. Bekanntlich war Mitte des Sommers 1829 der Dachstuhl bereits mehr oder weniger fertig gestellt. Bis zum Herbst 1831 war der größte Teil des Kirchdaches offenbar mit Kupferblech bedeckt. Der Bau des Holzgerüsts des Spitzhelms, der dem ehemaligen Dach im gotischen Stil ähnelte, fand in den Jahren 1833 und 1834 statt, aber seine Kupferbedeckung erhielt er erst im Jahre $1836 .{ }^{5}$

Auf dem Gemälde ist noch eine weitere Kirche dargestellt. Wahrscheinlich handelt es sich um eine der ersten Bilddarstellungen der orthodoxen Verklärung-des-Herrn-Kirche in ihrer heutigen Form mit einer Kuppel (Projekt 1827, Architekt Avraam Melnikov; Bauarbeiten von 1829 bis 1830 unter der Leitung des Architekten Johann Bantelmann). Es handelte sich um die ehemalige Kirche des Nonnenklosters der Zisterzienser, die während der schwedischen Zeit von der schwedischen lutherischen Gemeinde genutzt worden war, die aber nach dem Großen Nordischen Krieg auf Anordnung der russischen Staatsmacht in die Hände der orthodoxen Gemeinde überging. Aufgrund der Baurechnungen gefolgert, war die Kuppel mitsamt ihrem Unterbau aus Kalkstein und dem Tambour bereits Ende 1829 fertig gestellt. Der schmalere und höhere Glockenturm mit dem barocken Spitzhelm war schon im Jahre 1776 auf den Mauern des Treppenganges der früheren Klosterkirche errichtet worden, nun wurde aber der Spitzhelm etwas abgeändert. In dieser Form ist er auch bereits auf dem vorliegenden Gemälde zu sehen. Während der Klosterzeit verfügte die Kirche nur über einen kleinen Giebelturm. ${ }^{6}$ Das neue Aussehen der Kirche machte das auf dem Gemälde dargestellte Motiv sicherlich interessanter. Zusammenfassend gesagt: von den Informationen, die über die Kirchen bekannt sind, und von dem Todeszeitpunkt des Künstlers ausgehend, müsste das Bild aus den Jahren von 1830 bis 1831 stammen (unter der Voraussetzung, dass das Dargestellte weitgehend dem realen Zustand entsprach). Von dem, was der Künstler selbst in einem Brief knapp einen Monat vor seinem Tod äußerte, kann man entnehmen, dass

5 Angaben über die Wiederherstellung der Olaikirche bei: Ants Hein, „Taastamistööd Oleviste kirikus aastail 1828-1840", Eesti arhitektuuriloo küsimusi. Arhitektuuriajaloo sektsiooni I sügisseminar, Tallinn, 13.-14. okt. 1981. a. (Tallinn: Eesti NSV Ehituskomitee, 1981), 171-172.

6 Angaben über die Renovierungen der Verklärungskirche: Sulev Mäeväli, „Issanda Muutmise peakiriku remondid ja ümberehitused 18.-20. sajandini”, Vana Tallinn, IV (VIII) (Tallinn: 1994), 36-39. 
er fast bis zu seinen letzten Atemzügen schöpferisch aktiv war, indem er immer noch an den Krimansichten arbeitete und nach neuen Arten und Weisen suchte, wie er in seine Ansichten ein größeres Gefühl der Weite und der Freiheit einbringen könnte.

Dass das Bild in die letzten Lebensjahre des Künstlers gehört (falls es sich denn tatsächlich um ein Gemälde von Kügelgens handelt), bestätigen noch weitere Beobachtungen. Für die Stadtansichten, die auf ungefähr die gleiche Zeit datiert worden sind, ${ }^{8}$ stellt eine am Hintergrund des Meeres auf der Schonen-Bastion (schwedisch: bastionen Skåne oder Skånska bastionen, estnisch: Skoone bastion) emporragende riesige Fahnenstange, an der wehend zum Beispiel diverse Versionen der Flagge der russischen Marine mit dem Andreaskreuz dargestellt sind (offenbar als ein Kennzeichen von Reval als eine Seefestung), ein charakteristisches Element dar. In der Ferne im ufernahen Meer zieht sich als ein weißer Strich eine als Doppelbatterie bezeichnete Befestigung entlang, die in der Form, die hier zu sehen ist, in Grundzügen in den Jahren von ungefähr 1829 bis 1833 ausgebaut wurde. ${ }^{9}$

\section{DAS MOTIV}

Die Bezeichnung des Gemäldes ist auf Vereinbarungsbasis erfolgt, denn die Patkul-Treppe, die sich an der nordwestlichen Seite der Erhöhung des Dombergs befindet, wurde an der Stelle eines früheren Fußpfades erst Anfang des 20. Jahrhunderts gebaut. Ihren Namen erhielt sie von dem Patkul-Reduit und dem gleichnamigen Graben, die sich eben dort unten am Steilhang des Glints befanden, die wiederum wahrscheinlich nach dem schwedischen Vizegouverneur Estlands während des Großen Nordischen Krieges, Dietrich Friedrich von Patkul benannt worden waren. Früher wurde das hier zu besprechende Motiv normalerweise als „Ausblick vom Stenbock-Haus" bezeichnet. Dieses bemerkenswerte klassizistische Haus, in dem heute die Regierung der Republik Estland ihren Sitz hat, wurde Ende des 18. Jahrhunderts gebaut und es hat seinen Namen nach seinem Bauherren und Besitzer, dem Grafen Jakob Pontus

7 Peets, „Karl Ferdinand v. Kügelgen”, 81.

8 Zum Beispiel Johannes Hau, Suur Rannavärav Tallinnas (Die Große Strandpforte in Reval), ca 1820-1828, in der Sammlung des Tallinner Stadtmuseums (Tallinna Linnamuuseum, TLM); Jegor Grezdov, Tallinna vaade merelt (Aussischt auf Reval vom Meer aus), 1831, in der Sammlung des Estnischen Kunstmuseums.

9 Heino Gustavson, Tallinna vanemad merekindlused (17-19. sajand) (Tallinn: Olion 1994), 37-38. 
Stenbock erhalten. Sowohl die Treppe, das Haus als auch die populäre Aussichtsplattform sind auch heute noch erhalten.

Der erste bekannte Urheber einer ähnlichen Ansicht ist nicht von Kügelgen, sondern sein Zeitgenosse, der Revaler Künstler und hauptberuflicher Malermeister Johannes Hau (1771-1838), der überwiegend die Technik der Guasch einsetzte. Leider ist uns das Gemälde von Hau nur über die von Theodor Gehlhaar nach seinem Vorbild angefertigte Aquatinta bekannt, die in einer Reihe der Ansichten von Kügelgens und Haus erschien, die vom örtlichen Verleger und Buchhändler Georg Eggers im Jahre 1828 herausgegeben wurde..$^{10}$ Die Aquatinta wiederum stellte das Vorbild für die im Jahre 1833 in Paris bei Lemercier gedruckte Lithographie dar (eine Nachzeichnung von Edouard-Jean-Marie Hostein und Victor-Vincent Adam) ${ }_{,}^{11}$ bei der der Name des ursprünglichen Autors nicht mehr erwähnt wurde. Obwohl von Kügelgen die Ansicht Haus entweder im Original oder im Druck gesehen haben musste, handelte es sich offensichtlich um zwei komplett unterschiedliche Gemälde mit einem ähnlichen allgemeinen Motiv. Die Ansicht von Hau ist in Richtung der Vorstadt Fischermay um einiges breiter gewesen, weshalb die Zone der Erdbefestigungen darauf allgemeiner und zumindest auf den Gravüren auch völlig falsch dargestellt ist.

Das letzte mehr oder weniger in Betracht kommende Beispiel des gegebenen Motivs in der Kunst stammt aus dem Jahre 1854 in Form von einer Lithographie Ernst Hermann Schlichtings, der damals gerade als Zeichenlehrer an der örtlichen Domschule tätig war, und die eine Menschenmenge darstellt, die auf der Aussichtsplattform die Aktionen der englisch-französischen Marineeinheiten während der Seeblockade in der Revaler Bucht betrachtet. ${ }^{12}$ Nach dem Krimkrieg, im Jahre 1858 wurde ein Großteil der bis dahin bereits hoffnungslos veralteten Bastionszone Revals endlich für den zivilen Gebrauch freigegeben (darunter der auf dem Gemälde sichtbare Abschnitt mit dem Palmqvist-Reduit und

10 J. Hau del: Th. Gehlhaar sc. Vue prise de la maison de Steenbock, aus der Serie Collection de XII. vues. Gravées a l'aquatinte d'après Charles de Kügelgen et Johannes Hau par Th. Gehlhaar (1828). Vorhanden in der Sammlung des Tallinner Stadtmuseums.

11 E. Hostein Lith. fig. par V. Adam. Vue prise de la maison de Steinbock, Lithographie (Lemerciers Werkstatt, Paris), erschienen sowohl in der Serie Reval als auch als lose Blätter (1833). Verschiedene Versionen sind sowohl in der Sammlung des Estnischen Kunstmuseums als auch in der des Tallinner Stadtmuseums vorhanden.

12 Ernst Hermann Schlichting, Erinnerung an Reval im Sommer 1854 (Souvenir de Revale, en été 1854), Lithographie (Friedrich Wilhelm Macdonalds Werkstatt, Reval). Vorhanden in der Sammlung des Estnischen Kunstmuseums und des Tallinner Stadtmuseums. 
der Neuen Kontergarde). Im Jahre 1864 hatte man sich dafür entschieden, auch den Befestigungsabschnitt an der Meeresseite mitsamt dem größten einzelnen Verteidigungsbauwerk Revals, der Schonen-Bastion, aufzugeben. ${ }^{13}$ Nach dem Einebnen der Mehrheit der Wälle und nach der Auffüllung der Gräben wurde der Ausblick vom Stenbock-Haus aus weniger interessant und angenehm als bisher. Im Jahre 1870 wurde dorthin die Endstation der Eisenbahnverbindung zwischen St. Petersburg und Reval mitsamt der entsprechenden technischen Infrastruktur gebaut, der Baltische Bahnhof, und das Gebiet unmittelbar vor der Stadtmauer wurde am Anfang als Heuschlag, später als Ausstellungsplatz eingesetzt, heutzutage steht dort ein Park. So hat man es auf den späteren Ansichtspostkarten und auf den Kunstwerken bevorzugt, sich bei dieser Ansicht nur auf einen Teil der Altstadt zu beschränken.

\section{WAS IST AUF DEM GEMÄLDE DARGESTELLT WORDEN?}

Reval war während der Zeit unter der russischen Herrschaft (17101917) das Zentrum und die Hauptstadt des Gouvernements Estland (auch Ehstland, russisch: Ėstljandija, das heutige Nordestland). In militärischer Hinsicht war Reval vor allem eine Seefestung und neben Kronstadt eines der bedeutendsten Zentren der Baltischen Flotte des Reiches, aber im Falle einer Landung des Gegners sollte die Stadt bereit sein, auch einen Angriff von Marineinfanterie zu einem gewissen Grad zurückschlagen zu können, weshalb die Zone der Bastionen beibehalten wurde. Von der allgemeinen Lebensweise her blieb Reval im 19. Jahrhundert eine Provinzstadt, insbesondere im Vergleich mit Städten in der Nachbarschaft wie St. Petersburg, des sich schnell entwickelnden Helsinkis, der neuen Hauptstadt des Großfürstentums Finland, und dem historischen Zentrum der baltischen Gouvernements des Reichs, Riga.

Auf dem Gemälde von Kügelgens ist ein Teil der Unterstadt, ein Teil der Befestigungszone, die sie umgibt und in einer Ecke etwas von der Vorstadt zu sehen. Den Hintergrund bildet die Revaler Bucht und der weite Himmel, der die gesamte obere Hälfte des Bildes einnimmt. Wie wir sehen, waren für die Unterstadt eng gedrängte Häuser mit Wänden in hellen Tönen und - was aus diesem Blickwinkel besonderes ins Auge

13 Rudolf Kenkmaa, Gustav Vilbaste, Tallinna bastionid ja haljasalad (Tallinn: Eesti Raamat, 1965), 49. 
sticht - mit roten Dächern sowie mit weiß getünchten Schornsteinen charakteristisch, die Schornsteine waren dabei von unterschiedlicher Gestalt. Das Straßennetz, das in Reval als verhältnismäßig unregelmäßig erscheint, ist nicht besonders gut wahrnehmbar, aber eventuell macht eben diese scheinbare Zufälligkeit die Beobachtung von diesem Teil des Bildes interessant. Einige Häuser und Gärten sind auch schon auf dem engen Gebiet zwischen der mittelalterlichen Stadtmauer und dem Erdwall entstanden. Wie die Kunsthistorikerin Helmi Üprus anhand der Zeichnungen vorgeführt hat, die zum Zweck der Anfertigung eines Stadtmodells um das Jahr 1825 herum hergestellt worden waren, hatte Reval bis dahin im Großen und Ganzen sein mittelalterliches Aussehen einer alten Hansestadt beibehalten, wobei Kaufmannshäuser mit hohen dreieckigen Giebeln charakteristisch waren, die Richtung Straße blickten. Gleichzeitig nahm in den ersten Jahrzehnten des 19. Jahrhunderts, besonders seit den zwanziger Jahren der Druck von Seiten der Staatsmacht zu, die Fassaden durch das Einsetzen von Musterfassaden , auszuschmücken". Einen Gegensatz zur Unterstadt bildete der Domberg mit seinen Adelswohnsitzen, die über ein moderneres Aussehen verfügten. ${ }^{14}$ Auf dem Gemälde von Kügelgens erblicken wir in der Unterstadt nur einige einzelne Häuser, deren Traufen entlang der Straße verlaufen und deren Fenster regelmäßig untergebracht sind. Das augenfälligste von ihnen ist das gelbe Eckhaus Breite Straße 15 (estnisch: Lai tänav), dessen Architekt eine Koryphäe der klassizistischen Architektur war, nämlich Carl Ludwig Engel, der, bevor er als Entwerfer der Stadt Helsinki bekannt wurde, als Stadt-Baumeister von Reval gearbeitet hatte (real war er in diesem Beruf jedoch nur von 1809 bis 1814 tätig gewesen). ${ }^{15}$ Das ändert nichts an der Tatsache, dass es sich bei dem im Vordergrund des Bildes dargestellten Stadtteil - der Umgebung des ehemaligen Nonnenklosters der Zisterzienser - um einen verhältnismäßig peripheren Stadtteil handelte, worauf auch der Umstand hindeutet, dass sie von dem längsten Abschnitt der Stadtbefestigungen ohne ein einziges Stadttor umgeben wurde. Bei dem größeren, viereckigen Komplex, der links von der Verklärungskirche hinter der Stadtmauer zu vermuten ist, handelt es sich in der Tat um eine Klosterklausur in einer etwas umgebauten

14 Helmi Üprus, Tallinn aastal 1825 (Tallinn: Kunst, 1965).

15 Helmi Üprus, „Carl Ludwig Engel Tallinnas”, Töid kunstiteaduse ja -kriitika alalt, 3 (1983), 163. 
Gestalt, in der sowohl damals als auch heutzutage ein Gymnasium untergebracht war und ist.

Die hauptsächlich aus dem 14. und aus dem 15. Jahrhundert stammende Stadtmauer mitsamt ihren Türmen ist zum Zeitpunkt des Malens noch in einem verhältnismäßig guten Zustand gewesen. Im 18. und im 19. Jahrhundert war ein Teil der größeren Türme, die im Hinterland verblieben waren, als Depot für Schießpulver und andere militärische Ausrüstungsgegenstände eingesetzt worden, insbesondere wurden die unteren gewölbten Stockwerke genutzt. Eben deshalb verfügte offenbar die Mehrzahl von den auf dem Bild dargestellten runden Türmen noch über ein Dach (eine Ausnahme bildet der Süsternturm (estnisch: Nunnatorn)), wobei sie in vielen Fällen niedriger waren, als die bis heute wieder aufgebauten Türme. Beachtung verdient, dass bei dem vorderen kleinen viereckigen Turm, dem Badstubenturm, hier noch die obere Etage erhalten ist, die Ende des 19. Jahrhunderts auf Basis der freien Phantasie restauriert wurde. Auch ist der obere Teil von anderen Türmen und von der Stadtmauer später teilweise abgebröckelt. Ein Kenner könnte dem Maler eine teilweise etwas vereinfachte Darstellung der Schießscharten in Vergleich zur Wirklichkeit vorwerfen. Der Park vor der Stadtmauer trägt heute den Namen Platz der Türme (estnisch: Tornide väljak).

Die Zone der Erdbefestigungen Revals hatte bis zum Ende des 18. Jahrhunderts und dem Anfang des 19. Jahrhunderts ihren größten Umfang erreicht, aber ein stiller Niedergang hatte bereits eingesetzt. Bis zum Jahre 1830 stellten Befestigungsanlagen in Form von Bastionen im Militärwesen schon längst den Stand des gestrigen Tages dar. Die Verwirklichung der Pläne, die zur Erneuerung der Verteidigungseinrichtungen Revals und zum Übergang auf das moderne System der Forts zusammengestellt wurden, zögerte sich jedoch hinaus. Die von den Militäringenieuren erstellten Pläne der bestehenden Erdbefestigungszone sind meistens ziemlich allgemein gehalten und hinterlassen den Eindruck, als ob es sich um Verteidigungsbauten gehandelt habe, die in einem idealen Zustand waren, eine vollkommen geometrische Form hatten und über glatte, gut gepflegte Hänge verfügten. Über das Gemälde von Kügelgens erhalten wir eine vertrauenswürdigere Vorstellung von dem tatsächlichen Zustand der Erdbefestigungen. Im Allgemeinen fehlt ihnen kaum etwas, aber die Wälle und insbesondere die Schießscharten in den Brustwehren fangen jedoch bereits an, eine etwas unbestimmtere Gestalt anzunehmen. Die Ränder des Wallgrabens haben sich mit Gestrüpp bedeckt, 
irgendwo ist etwas von der Stützmauer abgebröckelt, irgendwo hat man den Weg mit Fußpfaden von den weiter reichenden Ecken des äußeren Befestigungswalls abgekürzt. Größere Bäume sind als Ausnahme nur auf der höchsten Stufe der Schonen-Bastion zu sehen, auf dem Kavalier, wo man bewusst eine Reihe von Linden so hingepflanzt hatte, so dass sie Schatten spenden und einen Augenschmaus bieten konnten, aber das Zielen oder die Bedienung der Kanonen nicht behinderten.

Sogar im Revaler Kontext kann der auf dem Gemälde von Kügelgens dargestellte südwestliche Abschnitt der Erdbefestigungen sich nicht einer besonderen militärischen Stärke rühmen. Seine Rolle bestand darin, einen möglichen Angriff zwischen dem Domberg und dem Meer zu stoppen, wobei die Befestigungen am Meer auch über eine gewisse Bedeutung vom Standpunkt des Küstenschutzes her verfügten. Bei der SchonenBastion, die in der Ferne zu sehen ist, handelt es sich einzeln gesehen zwar um einen prächtigen Bau, aber die Tatsache, dass die geplanten Nachbarbastionen nicht fertig gebaut wurden, verminderte ihren Wert und schränkte die Ausnutzung ihres Gesamtpotentials deutlich ein. Im Vordergrund sehen wir zwei Erdwälle hintereinander, aber der innere von ihnen hat eine wenig gegliederte Form und auch der äußere, obwohl mit einer dem bastionären System ähnlichen, gebrochenen Form, erinnerte eher an eine Außenbefestigungslinie und war offenbar auch als etwas Zeitweiliges geplant, bis sich die Möglichkeit zur Errichtung der Bastionen ergibt. Zugleich war die Landschaft der Umgebung verhältnismäßig eben, so dass sogar eine kleine Höhendifferenz den Verteidigern einen Vorzug verlieh. Wie es auf dem Bild ersichtlich wird, hat man es nicht übers Herz gebracht, den jahrhundertealten Koffer (siehe weiter unten), ebenso wie die Reduits und die Logements (hier in der Bedeutung bestimmter Kaponnieren), die während des Großen Nordischen Krieges im Laufe der Vorbereitungen für eine Belagerung in Eile gebaut worden waren, abzureißen. Man hat versucht, alles, was im Laufe unterschiedlicher Zeiten errichtet worden war, in ein notdürftiges und einheitliches Verteidigungssystem zu integrieren. An dieser Stelle würde es keinen Sinn ergeben, die allgemeine Logik dessen, wie ein bastionäres System funktioniert, oder auch den Werdegang der Erdbefestigungszone Revals nachzuerzählen. Die auf dem Bild dargestellten entsprechenden Elemente sind in der gemeinsamen Explikation der nebenan angeführten schematischen Zeichnung und des Auszugs des historischen Stadtplans genannt worden. 


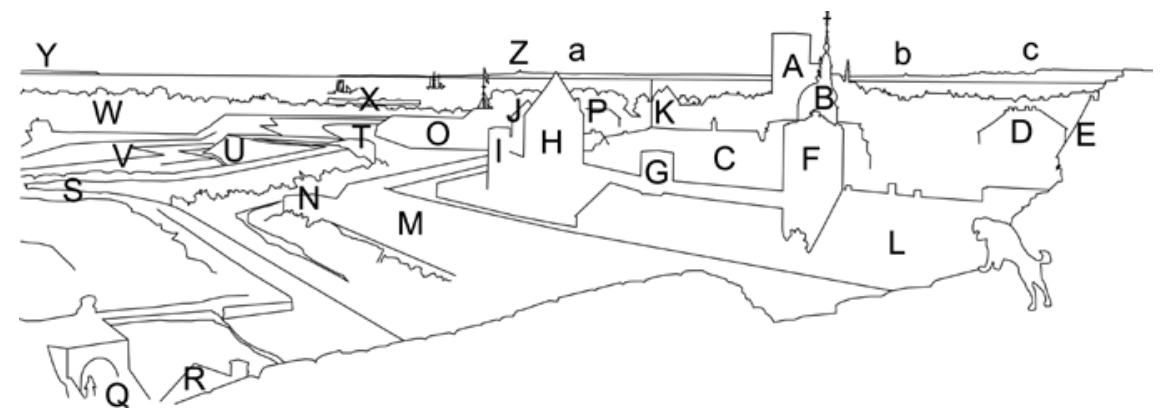

Abb. 2. Zeichnung von Ragnar Nurk

\section{Bauten der Unterstadt:}

A - Die Olaikirche (estnisch: Oleviste kirik).

B - Die orthodoxe Verklärung-des-Herrn-Kirche (in der ehemaligen,

umgebauten Kirche des Nonnenklosters der Zisterzienser).

C - Das Gymnasium (in der ehemaligen Klosterklausur).

D - Das Wohnhaus Breite Straße 15, Architekt Carl Ludwig Engel.

Die mittelalterliche Stadtmauer (mit den heutigen, teilweise historischen,

\section{teilweise neuen Namen):}

E - Der Torturm der Süsternpforte (estnisch: Nunnavärav).

F - Der Süsternturm (estnisch: Nunnatorn).

G - Der Badstubenturm (estnisch: Saunatorn).

H - Der Turm Goldener Fuß (estnisch: Kuldjala torn).

I - Der Turm hinter den Süstern (estnisch: Nunnadetagune torn).

J - Der Loewenschede-Turm.

K - Der Reeperbahn Turm (estnisch: Köismäe torn).

L _ - Die an die Stadtmauer errichteten Gebäude mitsamt den Gärten.

\section{Der innere Verteidigungswall:}

M - Der Nonnenwall (estnisch: Nunnavall).

N - Der Koffer (die Kaponniere) namens Mesekast (Mistkasten).

0 - Die Schonen-Bastion (schwedisch: bastionen Skåne oder Skånska bastionen,

estnisch: Skoone bastion).

P - Der Pulverspeicher der Schonen-Bastion.

\section{Der äußere Verteidigungswall:}

Q - Die zweite Wall pforte der Süsternpforte (estnisch: Nunnavärav).

R - Das Wächterhaus der Süsternpforte.

S - Das Palmqvist-Reduit.

T - Das Lieve-Logement (eine Kaponniere).

U - Die Neue Kontergarde;

V - Der zweite Wallgraben, der verdeckte Weg, der sich auf seinem äußeren

Ufer befand, und der Glaciswall (auf dem Weg Querwälle oder Traversen).

\section{Anderes:}

W - Die Vorstadt Fischermay (estnisch: Kalamaja).

$X \quad$ - Die Doppelbatterie.

Y - Die Insel Nargen (estnisch: Naissaar).

Z - Die Steilküste von Wiems (estnisch:Viimsi) mitsamt dem Leuchtturm.

a - Der Gutshof Wiems (estnisch:Viimsi).

b - Die Ruinen des Klosters St. Brigitten (estnisch: Pirita).

c - Die Zuckerfabrik und die Steilküste von Marienberg (estnisch: Maarjamäe pank).

d - Das Stenbock-Haus 


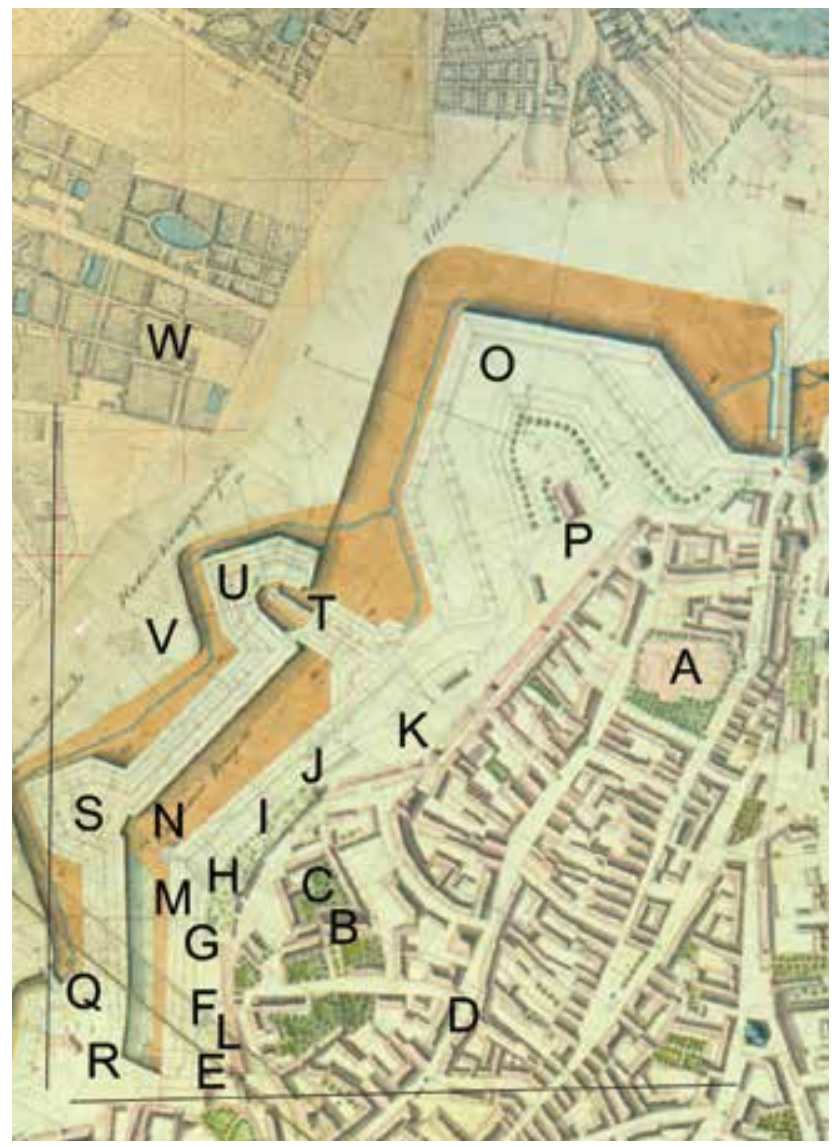

Abb. 3. Plan des Jahres 1825 (Fragment): Russisches Militärhistorisches Staatsarchiv (Rossijskij gosudarstvennyj voenno-istoričeskii archiv, RGVIA), Moskau, Bildbearbeitung von Ekke Lepp

Extra erwähnt werden sollte vielleicht nur der unmittelbar vor der Stadtmauer verlaufende Nonnenwall (estnisch: Nunnavall) mitsamt dem steinernen Verteidigungsbau, der sich in seiner Biegung ungefähr vor dem Turm der Goldene Fuß (estnisch: Kuldjala torn) befand. Bei den frühen, für die Kanonen angepassten Befestigungsanlagen und den frühen Bastionärbefestigungen in Alt-Livland (in allgemeinen Zügen das heutige Staatsgebiet von Estland und Lettland) handelt es sich eigentlich um ein bisher wenig erforschtes Thema, das im Rahmen des vorliegenden Beitrags nur markiert werden kann. Ein verdienstvoller Forscher der mittelalterlichen Stadtbefestigungen von Reval, der Architekt Rein Zobel, interpretierte diesen Wall in seinem letzten, noch zu seiner 
Lebenszeit erschienenen Werk (2011) als den ersten Verteidigungsbau der Renaissance in Reval, zu dem von Anfang an auch zwei „Steinbastionen des altitalienischen Systems" gehörten, die sich an den Biegungen des Walls befanden. ${ }^{16}$ Die anderen dieser sogenannten. Bastionen verblieben später innerhalb der Schonen-Bastion, die im Hintergrund der Gemälde zu sehen ist. In der 1980 erschienenen ursprünglichen Version dieser Monographie hatte er im Falle dieser angeblichen Bastionen nach dem Vorbild der früheren Autoren den Terminus „Koffer" verwendet, indem er sie für spätere, während des Livländischen Krieges (1558-1583) hinzugefügte Bauwerke hielt. ${ }^{17}$ In der neuen Version ließ Zobel nur die Möglichkeit offen, dass die endgültige Form dieser „,ersten Bastionen von Reval" aus einer etwas späteren Zeit stammen könnte. ${ }^{18}$ Das Gemälde Kügelgens ist mit hoher Wahrscheinlichkeit die wirklichkeitsgetreueste Darstellung des Nonnenwalls. Indem er sich auf die Angaben der mittelalterlichen Rechnungsbücher Revals ${ }^{19}$ stützte, datierte Zobel die Gründung des Nonnenwalls auf die Jahre von 1463 bis 1472, was begreiflicherweise für die Bastionen oder überhaupt für irgendwelche bastionsförmigen neuzeitlichen Verteidigungsbauten ein sehr frühes Datum wäre, insbesondere außerhalb Italiens. ${ }^{20}$ Deshalb muss betont werden, dass es sich zum jetzigen Standpunkt im Licht der bekannten Fakten nur um eine Fiktion handelt, wenn man die zu betrachtenden zwei Steinbauten für gleichzeitige Bauwerke mit dem Nonnenwall hält.

16 Rein Zobel, Tallinn (Reval). Keskaegsed kindlustused (Tallinn: Eesti Kunstiakadeemia, 2011), 246-248.

17 Rein Zobel, Tallinna keskaegsed kindlustused (Tallinn: Valgus, 1980), 256. Früher beispielsweise: Kenkmaa, Vilbaste, Tallinna bastionid, 22.

18 Zobel, Tallinn (Reval), 248.

19 Die Historikerin Epp Siimo hat die mittelalterlichen Rechnungsbücher Revals transkribiert und, mit Kommentaren und Verzeichnissen versehen. Die entsprechenden Manuskripte befinden sich in der Baltica-Abteilung der Akademischen Bibliothek der Universität Tallinn.

20 Eine Bastion in der richtigen Bedeutung des Wortes bildete sich wahrscheinlich zum Ende des 15. Jahrhunderts und am Anfang des 16. Jahrhunderts in Mittelitalien heraus. Versuche mit Türmen, die wie eine Bastion aussahen, wurden dort allerdings schon seit den vierziger bis sechziger Jahren des 15. Jahrhunderts unternommen. In der zweiten Hälfte des 15. Jahrhunderts waren jedoch noch jene bastionsähnlichen Türme mit runden Formen eher verbreitet. Zu einer allgemein anerkannten Norm sowohl bei den Forts als auch bei den Stadtbefestigungen war die Bastion erst bis zu den dreißiger Jahren des 16. Jahrhunderts geworden. Die Erfindung der Bastion kann keinem bestimmten Architekten zugeschrieben werden, obwohl solche Behauptungen auch aufgestellt worden sind. Wichtiger als die Rolle der Theoretiker war dabei die der Praktiker, der praktisch tätigen Architekten und der Baumeister. Gleichzeitig haben auch andere Orte und Völker einen Anspruch auf die Ehre der Erfindung der Bastion erhoben, wie zum Beispiel die entsprechenden Hypothesen über die Türkei, über Böhmen und über die Insel Rhodos belegen (siehe näher John Rigby Hale, „The Early Developement of the Bastion: an Italian Chronology c. 1450-c. 1534", Europe in the Late Middle Ages, eds. John Rigby Hale, John Roger Loxdale Highfield, Beryl Smalley (London: Faber, 1965), 466-494). 
In den Eintragungen der Rechnungsbücher ist nur unbestimmt die Rede vom Bau von Mauern und Gräben im Abschnitt der gegebenen Befestigungen: zwischen der Süsternpforte (estnisch: Nunnavärav) und der Großen Strandpforte (estnisch: Suur Rannavärav). Es haben bisher keine archäologischen Ausgrabungen stattgefunden, obwohl die Eskarpemauern unter der Erde wahrscheinlich erhalten geblieben sind. Es gibt wenigstens ein gewichtiges Argument, um den Nonnenwall mit den Ideen der Renaissance in Verbindung zu bringen, nämlich seine völlig regelmäßige Planung, wie Zobel in seinem neueren Werk betont. Tatsächlich, der Bau verfolgte in keiner Weise die Form der mittelalterlichen Stadtmauer, sondern bestand gemäß den aus dem Zeitraum vom 17. bis zum 19. Jahrhundert stammenden historischen Plänen aus zwei kürzeren Endabschnitten gleicher Länge, die sich an einen längeren mittleren Abschnitt unter gleichen Winkeln anschlossen (die Gesamtlänge betrug ungefähr 700 Meter). ${ }^{21}$

Ein weiterer Erforscher der Bastionen, der Historiker Rudolf Kenkmaa, hat in Bezug auf die zwei Bauten des Nonnenwalls die Bezeichnungen Koffer und Kaponniere verwendet. Laut seinen Angaben hat man den einen Bau, den von Kügelgen auf seinem Gemälde dargestellt hat, als Mesekast (Mistkasten) bezeichnet, und das andere Bauwerk, das sich ungefähr an der Stelle der Schonen-Bastion befand, etwas nobler als Louwenborch (Löwenburg). ${ }^{22}$ Die Bezeichnung Koffer (französisch coffre) tritt auch auf historischen Zeichnungen auf. ${ }^{23}$ Eventuell könnte man tatsächlich anstatt über Bastionen von einer Seelenverwandtschaft mit jenen Grabenverteidigungsanlagen sprechen, die der bekannte italienische Architekt Francesco di Giorgio Martini (1439-1502) als capannati bezeichnet hatte und auf seinen Zeichnungen entweder polygonal oder

21 Zobel, Tallinn (Reval), 247.

22 Kenkmaa, Vilbaste, Tallinna bastionid, 21-22.

23 Eine der Leitern der Befestigungsarbeiten, Gebhard Himsel, hat auf seiner Projektzeichnung von 1671 diese zwei Gebäude als „Katze im Graben” bezeichnet (Tallinna Linnaarhiiv (Tallinner Stadtarchiv, TLA), 230.1. Be 21.5). Auf den aus der schwedischen Zeit stammenden Plänen vom Ende des 17. Jahrhunderts bis zum Anfang des 18. Jahrhunderts stehen die Bezeichnungen Koffer (im Original couffert) und (Graben-) Kasematte (casemate, cassematten i grafwen) (Krigsarkivet (Schwedisches Kriegsarchiv, KrA), 0406:28:040:008 und 0406:28:040:023). Die Bezeichnung „Kasematte” bezieht sich eventuell auf die bombensicheren Räume innerhalb der Koffern. Auf den aus der russischen Zeit um die Wende des 18. und des 19. Jahrhunderts stammenden Plänen der Revaler Bastionszone ist das auf dem Gemälde abgebildete Gebäude einfach als „ein Rondell aus Stein” (russisch: Kammennoj Rondel') bezeichnet worden (zum Beispiel auf den Plänen aus den Jahren 1797 und 1825, für die Letztere siehe die Reproduktion: Üprus, Tallinn aastal 1825, Tafel XII), wobei das Rondell wahrscheinlich als eine allgemeine Bezeichnung im Sinne irgendeiner veralteten Befestigungsanlage erscheint. 
rund dargestellt hatte, meistens oben durch ein festes Dach bedeckt. ${ }^{24}$ Bei ihnen handelte es sich, worauf auch der Name hindeutet, doch eher um die Vorgänger der Kaponniere oder der Grabenwehr ${ }^{25}$ als den der Bastion. Beim Koffer handelt es sich um eine Unterart der Kaponniere, welche von seinem Aussehen her an ein Gepäck oder an einen Kasten erinnert haben könnte. Im gegebenen Fall handelt es sich offenbar um eine ausgesprochen realistische Begriffsbestimmung. Auf dem Gemälde von Kügelgen ist die Tatsache wahrzunehmen, dass (mindestens in der endgültigen Ausführung dieser Wehranlage) die Feuerkraft der Verteidiger hauptsächlich auf den geraden Wallabschnitten gezielt worden war, wobei die Koffern dahingegen den unmittelbar davorliegenden Wallgraben unter Beschuss genommen hatten, da dieser Wallgraben von den Wällen aus anderweitig nicht befriedigend zu schützen war. Mit der zuletzt beschriebenen Aufgabe wird der eigentliche Entstehungsgrund der Kaponniere ausgedrückt. Mit der Entwicklung des Bastionalsystems entstand die Möglichkeit, flankierendes Feuer aus den Nachbarbastionen zu eröffnen, und somit hatte sich dieses Problem gelöst.

Es ist interessant zu notieren, dass die Idee aus Reval auch weitergetragen werden konnte (offenbar nicht umgekehrt). Derartige Koffer, die vom Grundriss her einer Bastion ähneln und auch ähnliche Masse haben, sind auf den Plänen aus dem 17. Jahrhundert zweier westestnischer Burgen (Hapsal, estnisch Haapsalu, und Leal, estnisch Lihula) dargestellt. Am Ende des Mittelalters gehörten diese beide Burgen dem Bistum von Ösel-Wiek (estnisch: Saare-Lääne), wurden zerstört und haben ihre Bedeutung im Folge des Livländischen Krieges (1558-1583) verloren, wodurch man den terminus ante quem festlegen kann. In der Burg von

\footnotetext{
24 Eric Langenskiöld, „Bastionssystemets upprinnelse och tidigare utveckling: en studie över befästningskonsten i Europa fram till Vaubans skede”, Kungl. Vitterhets Historie och Antikvitets Akad. Handl, 55. Antikvariska Studier, 1 (1944), 281-282, fig. 29.

25 Hier werden die Begriffe „die Kaponniere” und „der Koffer” im Sinne einer Grabenwehr verwendet: ein ein- oder mehrstöckiger, schusssicherer und verteidigungsfähiger Hohlbau im Graben einer Festung, senkrecht zum Verlauf des Grabens errichtet und zur Grabenbestreichung dienend. Nicht zu verwechseln ist er mit anderen möglichen Bedeutungen. Dieselbe Begriffe bezeichnen auch gewisse Bestandteile der Fortifikationen des 19. Jahrhunderts, die oft auf die gleiche Art und Weise zum Schutz des Grabens bzw. des Vorfelds des Hauptwalls dienten von der Architektur her aber bedeutende Unterschiede zu den hier beschriebenen Objekten besassen. (Comité International d'Histoire de l'Art, Glossarium Artis 7: Festungen: der Wehrbau nach Einführung der Feuerwaffen. Systematisches Fachwörterbuch, 2, vollständig neu bearb. und erw. Aufl. (München, London, New York, Paris: K. G. Saur, 1990), 106, 114, 120-121; Elmar Brohl, Michael Losse, „Kaponniere”, Wörterbuch der Burgen, Schlösser und Festungen, hrsg. von Horst Wolfgang Böhme, Reinhard Friedrich, Barbara SchockWerner (Stuttgart: Philipp Reclam jun. 2004), 164).
} 
Hapsal, das eines der bedeutendsten Machzentren des Bistums war, ist ein Koffer an der rechtwinkligen Ecke des Burgwalles zu sehen. ${ }^{26}$ In der Burg Leal, die als Burg kleiner gewesen war, eigentlich aber als ein Kondominium unter der gemeinschaftlichen Herrschaft des Bischofs und des Livländischen Ordens stand, war eine dem Revaler Nonnenwall ähnliche Situation vorhanden - mit einem Wall, an den zwei Koffer angeschlossen waren. ${ }^{27}$

Vor dem Hintergrund des allgemeinen Werdegangs der Revaler Befestigungen bleibt der Nonnenwall auf jeden Fall eine schwer erklärbare Anomalie, denn weiterhin verlief alles nach einem ganz gewöhnlichen Entwicklungsweg: bis ungefähr zum Livländischen Krieg baute man weiterhin gedanklich noch im Mittelalter verhaftete Geschütztürme mit einer runden Verteidigungsfront (zum Teil sogar noch mit Maschikulis, die für die Vertikalverteidigung vorgesehen waren) und mit ihnen verbundene Vorwälle in unbestimmter Gestalt. Die Revaler Wehranlagen haben während des Livländischen Krieges zweimal (1570-1571 und 1577) eine Belagerung durch das Heer Ivans IV. widerstanden, wobei der Chronist Balthasar Russow, der beide Belagerungen miterlebt hatte, in seiner Chronik wiederholt betonte, dass die Stadt so gut durch die Wälle umgeben war, dass der Feind keinen Schuss direkt auf die Mauern schießen konnte. ${ }^{28}$ Hingegen wird von dem Chronisten anderswo bemerkt, dass Reval vor dem Krieg nicht in solcher Stärke befestigt gewesen war und erst am Beginn des Krieges angefangen wurde, Rondellen, Wälle, Mauern, Gräben und Streichwehren (Rundelen, Wellen, Muren, Grauen unde Stryckweren) zu bauen - die letzteren können etwas Kofferähnliches

$26 \mathrm{KrA}$, 0406:28:015:001 (Reproduktion: Kalvi Aluve, Eesti keskaegsed linnused (Tallinn Kirjastus: „Valgus”, 1993), Ill. 23; die Umzeichnung: Armin Tuulse, Die Burgen in Estland und Lettland (Dorpat: Dorpater Estnischer Verlag, 1942), Abb. 122); Schwedisches Reichsarchiv (Riksarkivet, RA), 81001:0485:00001 (Reproduktion: Aluve, Eesti keskaegsed, Ill. 19).

27 KrA, 0406:28:024:001 (Reproduktionen: Tuulse, Die Burgen, Abb. 26; Aluve, Eesti keskaegsed, Ill. 3).

28 Balthasar Russow, Chronica.der Provintz Lyfflandt (Barth: A. Seitner, 1584), 98v, 101. Volltext im Digitalarchiv der Estnischen Nationalbibliothek: http://digar.nlib.ee/digar/show/?id=104675 (Zuletzt aufgerufen 12.11.2013). 
gewesen sein. ${ }^{29}$ Eigentlich wird der Nonnenwall nicht von Russow explizit erwähnt, die Vermutung aber, dass dieser im Laufe des Krieges ergänzt wurde, kann der Wahrheit nahe liegen. Es ist nicht auszuschließen, dass es sich bei den Ergänzungen unter anderem einen Anbau von Koffern handelte. Die Bedeutung des Nonnenwalls für die Zeitgenossen wird auch durch die Tatsache betont, dass er eine prominente Stellung auf den früheren, aus dem 17. Jahrhundert stammenden Stadtansichten von Reval einnimmt. ${ }^{30}$

Wenn man nun von diesem Detail zum Schluss noch zum Gemälde von Kügelgens zurückkehrt, sehen wir links oben ein Stückchen der im Grünen versinkenden Vorstadt Fischermay. Es handelte sich ursprünglich offensichtlich wie der Name sagt um eine Siedlung von Fischern. Im Mittelalter wohnten dort sowohl Menschen estnischer Herkunft als auch Personen finnischer und der schwedischer Abstammung. Bis zum Anfang des 19. Jahrhunderts war Fischermay zum Teil auch zum Standort von den Nutzgärten einiger Stadtbürger und von einigen ruhigen Sommerhäusern geworden. Die Anforderungen einer Esplanade brachten Einschränkungen für die Errichtung von Bauten und ihren Charakter mit sich - Steingebäude waren deshalb nicht erlaubt. Bei der Ansicht von Kügelgens ist es sogar einigermaßen erstaunlich, so viele Häuser und Bäume zu sehen - offenbar handelte es sich um Obstgärten. Aus den Plänen wird zusätzlich noch ersichtlich, dass es dort viele Teiche gab. Die Häuser waren niedrig und einfach gebaut. Rote Schindeldächer haben sich nur wenige leisten können.

Im Hintergrund bleibt die Bucht von Reval. Am meisten Aufmerksamkeit erzielt die bereits erwähnte, aufsteigende Doppelbatterie. Über der Altstadt kann man die Hafenbauten eher erahnen als wirklich sehen. Am Horizont hinten links sehen wir undeutlich die Insel Nargen (estnisch: Naissaar), rechts das gegenüberliegende Ufer der Bucht mit

29 In der modernen Terminologie: „Streichwehr - eine Anlage zur Abgabe von Flankierungsfeuer” (Comité, Glossarium Artis, 148). In der estnischen Übersetzung sind fälschlicherweise „Bastionen” genannt (Balthasar Russow, Liivimaa kroonika, übersetzt von Dagmar und Hermann Stock (Tallinn: Hotger, 1993), 119). Dabei wird von Russow öfters die Bezeichnung „das Bollwerk” verwendet, offenbar im Sinne irgendeiner provisorischer Befestigung. Einmal kommt „Posteide” vor (es bleibt offen, ob es sich um eine Bastei bzw. ein Rondell oder eine Bastion handelt), das Wort ist aber eher als rhetorische Redewendung zu verstehen, wenn der Chronist berichtet, wie die Bürger der Nachbarstadt Dorpat (estnisch: Tartu) ihre Befestigungen vernachlässigt hatten („So war dar noch wall/noch Posteide/ noch jenich Dwenger gebuwet”; Russow, Chronica, 1584, 43v).

30 Zum Beispiel eine weitbekannte und mehrmals publizierte Anicht von Reval bei Matthäus Merian, die später auch Adam Olearius als Vorbild verwendet hat (beide sind Kupferstiche), und eine Bemalung auf der Stadtprivilegientruhe, die zur Zeit im Revaler Stadtmuseum aufbewahrt wird. 
der Halbinsel Wiems (estnisch: Viimsi) und mit St. Brigitten (estnisch: Pirita). Bei näherer Betrachtung sehen wir auch dort Bauwerke und Gebäudekomplexe, die tatsächlich existiert haben: den Leuchtturm und den Gutshof von Wiems, die Ruinen des Klosters St. Brigitten und die Zuckerfabrik. Auch das Relief ist erkennbar: die Kalksteinsteilküste in Wiems und in Marienberg (estnisch: Maarjamäe).

Ragnar Nurk: A view on talllinn with her fortifications Keywords: Karl (Carl) Ferdinand von Kügelgen (1772-1831); CitysCAPE, REALisM, TALLinN, First HALF OF THE $19^{\text {Th }}$ CENTURY, ARTILLERY FORTIFICATION

\section{SUMMARY:}

The article introduces one of the best examples of the once popular Tallinn cityscape motif: a painting that is artistically significant but also valuable as a historical document. The oil painting from the the collection of the Art Museum of Estonia is attributed to Karl (Carl) Ferdinand von Kügelgen (1772-1831), a landscape painter from Rhineland who had family ties in the Tallinn region and spent the last years of his life there. Besides that fact, the technical mastery also supports the attribution to Kügelgen. The representation is historically accurate - true to the period of time when Kügelgen resided in the city, and the detail of depictions implies substantial knowledge of the Tallinn area. Still, art historian Voldemar Vaga has drawn attention to the fact that in contrast to Kügelgen's typically Classicist and slightly beautifying depictions of reality, this painting is Realist to the smallest detail. The motif under scrutiny is common both in painting and in graphic art from the $1820 \mathrm{~s}$ to 1850 s.

The paintings with this motif depict a declining provincial city and a fort of the Russian Empire that had reached its height in the medieval times. Kügelgen's painting shows clearly the expanse of the entrenchments taking up the space between the city centre and the outskirts and it also exposes a dissimilarity in construction methods as well as a spatial separation between the two areas. An outstanding depicted detail is 
Nunnavall (German „Nonnenwall”), an earthen entrenchment seen just in front of the city wall together with a polygonal stone construction between the sections of the entrenchment - the most realistic extant depiction of this feature. From other images and the city plans of the time we can infer that initially there were two stone constructions - placed at both intersections of the entrenchment. One of the constructions is later incorporated into the larger Skoone bastion that can be seen on the background of the painting. The depiction of these buildings suggests that they functioned as caponiers - a type of a bunker for the defence of the moat, and not as bastions. Also, it is possible that these buildings were actually built later than the rest of the rampart, e.g. from the period of the Livonian War (1558-1583).

Tody The Old Town and its surrounding city wall have survived much the same as depicted in the painting. In contrast, the earthen entrenchments have been entirely flattened and in their place parks have been developed.

CV:

Ragnar Nurk is a doctoral student at the Institute of History of Tallinn University, and works as an archaeologist. His master's thesis on the development and architecture of a section of Tallinn bastion zone near Anthony's Hill (German „Antonius-Berg”, Estonian „Tõnismäe”) earned him the Estonian Academy of Sciences Student Research Prize in 2011. 

